\title{
DECLINING INCIDENCE OF INFANTILE HYPERTROPHIC STENOSIS (IHPS) IN GERMANY
} 2000-2008

\author{
J. Enders ${ }^{1}$, S. Turial ${ }^{2}$, F. Schier ${ }^{2}$, K.-P. Zimmer ${ }^{1}$, M. Heckmann ${ }^{1}$ \\ ${ }^{l}$ General Pediatrics and Neonatology, University of Giessen, Giessen, ${ }^{2}$ Pediatric Surgery, University of \\ Mainz, Mainz, Germany
}

Background: The incidencce of infantile hypertrophic pyloric stenosis (IHPS) is highly variable over time and regionally different. There have been reports of declining incidence in Sweden, the US, Denmark and Scotland. In Sweden, the decline observed was parallel to the declining incidence of Sudden Infant Death Syndrome (SIDS) and was attributed to campaigns against prone sleeping position.

Aim: How high was the incidence of IHPS in the federal states (n=16) of Germany 2000-2008? Did the incidence of IHPS correlate with incidence of SIDS?

Methods: Data were extracted from the public report of Health (Gesundheitsberichterstattung des Bundes). The number of IHPS (ICD 10 40.0) and SIDS (R95) the number of life birth (Male/Female) were collected in every federal state for the years 2000-2008.

Results: There was a decline in IHPS Incidence in Germany from 2000 (3.2086/1000LB [Range 1.67-5.33]) to $2008(2.0175 / 1000 \mathrm{LB}$ [1.74-3.72]). The recorded incidence was highly variable in different federal states and over time.

Furthermore, a decline in SIDS incidence was noted. The regional distribution of the incidence of SIDS was different from the distribution of the incidence of IHPS.

Conclusion: Incidence of IHPS declined about 38\% nationwide. A parallel decline in SIDS displayed a different pattern in regional distribution making a common cause less plausible. 